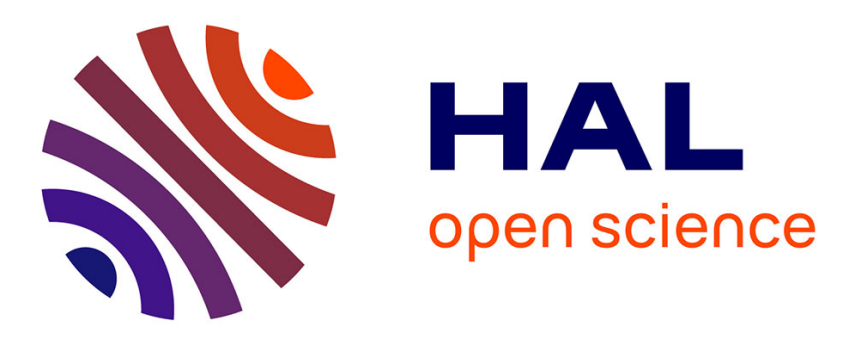

\title{
Identification of the delay parameter for nonlinear time-delay systems with unknown inputs
}

\author{
Gang Zheng, Jean-Pierre Barbot, Driss Boutat
}

\section{To cite this version:}

Gang Zheng, Jean-Pierre Barbot, Driss Boutat. Identification of the delay parameter for nonlinear time-delay systems with unknown inputs. Automatica, 2013, 49, 6, pp.1755-1760. 10.1016/j.automatica.2013.02.020 . hal-00817331

\section{HAL Id: hal-00817331 \\ https://inria.hal.science/hal-00817331}

Submitted on 8 Mar 2017

HAL is a multi-disciplinary open access archive for the deposit and dissemination of scientific research documents, whether they are published or not. The documents may come from teaching and research institutions in France or abroad, or from public or private research centers.
L'archive ouverte pluridisciplinaire HAL, est destinée au dépôt et à la diffusion de documents scientifiques de niveau recherche, publiés ou non, émanant des établissements d'enseignement et de recherche français ou étrangers, des laboratoires publics ou privés. 


\title{
Identification of the delay parameter for nonlinear time-delay systems with unknown inputs
}

\author{
G. Zheng ${ }^{a, \mathrm{~b}}$, J.-P. Barbot ${ }^{a, \mathrm{c}}$, D. Boutat ${ }^{\mathrm{d}}$ \\ ${ }^{a}$ INRIA Lille-Nord Europe, 40 avenue Halley, 59650 Villeneuve d'Ascq, France \\ ${ }^{b}$ LAGIS CNRS 8219, Villeneuve dAscq 59651, France \\ ${ }^{c}$ ECS ENSEA, 6 Avenue du Ponceau, 95014 Cergy-Pontoise, France \\ ${ }^{d}$ Loire Valley University, ENSI-Bourges, 88 Bd. Lahitolle, 18020 Bourges Cedex, France.
}

\begin{abstract}
By using the theory of non-commutative rings, this paper studies the delay identification of nonlinear time-delay systems with unknown inputs. A sufficient condition is given in order to deduce an output delay equation from the studied system. Then necessary and sufficient conditions are proposed to judge whether the deduced output delay equation can be used to identify delay involved in this equation. Two different cases are discussed for the dependent and independent outputs, respectively. The presented result is applied to identify delay in a biological system.
\end{abstract}

Keywords: Time-delay system, Delay identification

\section{Introduction}

Time-delay systems are widely used to model concrete systems in engineering sciences, such as biology, chemistry, mechanics and so on (Kolmanovskii and Myshkis (1999); Niculescu (2001); Richard (2003)). Many results have been reported for the purpose of stability analysis, by assuming that the delay of the studied systems is known. And it makes the delay identification one of the most important topics in the field of time-delay systems.

Up to now, various techniques have been proposed for the delay identification problem, such as identification by using variable structure observers in Drakunov et al. (2006), by a modified least squares technique in Ren et al. (2005), by convolution approach in Belkoura (2005), by using the fast identification technique proposed in Fliess and Sira-Ramirez (2004) to deal with online identification of continuous-time systems with structured entries in Belkoura et al. (2009) and so on.

Recently, Anguelova and Wennberg (2008) proposed to analyze the delay identification for nonlinear control systems with a single unknown constant delay by using the non-commutative rings theory, which has been applied to analyze nonlinear timedelay systems firstly by Moog et al. (2000) for the disturbance decoupling problem of nonlinear time-delay system, and for observability of nonlinear time-delay systems with known inputs by Xia et al. (2002) and with unknown inputs by Zheng et al. (2011). Although the inputs are commonly supposed to be known and are usually used to control the studied system, however there exists as well other cases, such as observer design for time-delay systems, in which the inputs can be unknown (Sename (1994); Darouach et al. (1994); Koenig et al. (2005); Yang and Wilde (1988)). Moreover, some proposed unknown inputs observer design methods do depend on the known delay, which should be identified in advance. Motivated by this requirement and inspired by the work of Anguelova and Wennberg (2008), this paper investigates the delay identification problem for nonlinear time-delay systems with unknown inputs.

This paper is organized as follows. Section 2 recalls the algebraic framework proposed in Xia et al. (2002). Notations and definitions are given in Section 3. Necessary and sufficient conditions are discussed for identifying the delay from only the outputs of systems in Section 4. Main theorem for the case where the outputs are independent over the non-commutative rings is stated in Section 5, and the proposed result is applied to identify delay of biological system in Section 6.

\section{Algebraic framework}

Denote $\tau$ the basic delay, and assume that the delays are multiple times of $\tau$. Consider the following nonlinear time-delay system:

$$
\left\{\begin{array}{l}
\dot{x}=f(x(t-i \tau))+\sum_{j=0}^{s} g^{j}(x(t-i \tau)) u(t-j \tau) \\
y=h(x(t-i \tau))=\left[h_{1}(x(t-i \tau)), \ldots, h_{p}(x(t-i \tau))\right]^{T} \\
x(t)=\psi(t), u(t)=\varphi(t), t \in[-s \tau, 0]
\end{array}\right.
$$

where $x \in W \subset R^{n}$ denotes the state variables, $u=$ $\left[u_{1}, \ldots, u_{m}\right]^{T} \in R^{m}$ is the unknown input, $y \in R^{p}$ is the measurable output, $i \in S_{-}=\{0,1, \ldots, s\}$ is a finite set of constant delays, $f, g^{j}$ and $h$ are meromorphic functions ${ }^{1}, f(x(t-i \tau))=$ $f(x, x(t-\tau), \ldots, x(t-s \tau))$ and $\psi:[-s \tau, 0] \rightarrow R^{n}$ and $\varphi:$ $[-s \tau, 0] \rightarrow R^{m}$ denote unknown continuous functions of initial

\footnotetext{
${ }^{1}$ means quotients of convergent power series with real coefficients (Conte
} et al. (1999); Xia et al. (2002)). 
conditions. In this work, it is assumed that for initial conditions $\psi$ and $\varphi$ system (1) admits a unique solution.

Based on the algebraic framework introduced in Xia et al. (2002), let $\mathcal{K}$ be the field of meromorphic functions of a finite number of the variables from $\left\{x_{j}(t-i \tau), j \in[1, n], i \in S_{-}\right\}$. For the sake of simplicity, we introduce delay operator $\delta$, which means

$$
\delta^{i} \xi(t)=\xi(t-i \tau), \xi(t) \in \mathcal{K}, \text { for } i \in Z^{+}
$$

and

$$
\begin{aligned}
\delta^{i}(a(t) d \xi(t)) & =\delta^{i} a(t) \delta^{i} d \xi(t) \\
& =a(t-i \tau) d \xi(t-i \tau)
\end{aligned}
$$

for $i \in Z^{+}$.

Let $\mathcal{K}(\delta$ ] denote the set of polynomials of the form

$$
a(\delta]=a_{0}(t)+a_{1}(t) \delta+\cdots+a_{r_{a}}(t) \delta^{r_{a}}
$$

where $a_{i}(t) \in \mathcal{K}$. The addition in $\mathcal{K}(\delta]$ is defined as usual, but the multiplication is given as

$$
a(\delta] b(\delta]=\sum_{k=0}^{r_{a}+r_{b}} \sum_{i+j=k}^{i \leq r_{a}, j \leq r_{b}} a_{i}(t) b_{j}(t-i \tau) \delta^{k}
$$

Differentiation of a function $h_{j}(x(t-i \tau))$ is defined as follows:

$$
\dot{h}_{j}(x(t-i \tau))=\sum_{i=0}^{s} \frac{\partial h_{j}}{\partial x(t-i \tau)} \delta^{i} f
$$

With the definition of $\mathcal{K}(\delta]$, (1) can be rewritten in a more compact form as follows:

$$
\left\{\begin{array}{l}
\dot{x}=f(x, \delta)+G(x, \delta) u=f(x, \delta)+\sum_{i=1}^{m} G_{i} u_{i}(t) \\
y=h(x, \delta) \\
x(t)=\psi(t), u(t)=\varphi(t), t \in[-s \tau, 0]
\end{array}\right.
$$

where $f(x, \delta)=f(x(t-i \tau))$ and $h(x, \delta)=h(x(t-i \tau))$ with entries belonging to $\mathcal{K}, u=u(t)$, and $G(x, \delta)=\left[G_{1}, \cdots, G_{m}\right]$ with $G_{i}=\sum_{j=1}^{n}\left(\sum_{l=0}^{s} g_{i}^{l} \delta^{l}\right) \frac{\partial}{\partial x_{j}}$.

Denote $\mathcal{M}$ the left module over $\mathcal{K}(\delta]$ :

$$
\mathcal{M}=\operatorname{span}_{\mathcal{K}(\delta]}\{d \xi, \xi \in \mathcal{K}\}
$$

where $\mathcal{K}(\delta$ ] acts on $d \xi$ according to (2) and (3). Note that $\mathcal{K}(\delta]$ is a non-commutative ring, however it is proved that the ring $\mathcal{K}(\delta$ ] is a left Ore ring (Ježek (1996); Xia et al. (2002)), which enables to define the rank of a module over this ring.

With the standard differential operator $d$, define the vector space $\mathcal{E}$ over $\mathcal{K}$ :

$$
\mathcal{E}=\operatorname{span}_{\mathcal{K}}\{d \xi: \xi \in \mathcal{K}\}
$$

which is the set of linear combinations of a finite number of elements from $d x_{j}(t-i \tau)$ with row vector coefficients in $\mathcal{K}$. Since the delay operator $\delta$ and the standard differential operator are commutative, thus the one-form can be written as $\omega=\sum_{j=1}^{n} a_{j}(\delta] d x_{j}$, where $a(\delta] \in \mathcal{K}(\delta]$. For a given vector field $\beta=\sum_{j=1}^{n} b_{j}(\delta] \frac{\partial}{\partial x_{j}}$ with $b_{j}(\delta] \in \mathcal{K}(\delta]$, the inner product of $\omega$ and $\beta$ is defined as follows:

$$
\omega \beta=\sum_{j=1}^{n} a_{j}(\delta] b_{j}(\delta] \in \mathcal{K}(\delta]
$$

with $\omega \in \mathcal{M}$.

\section{Notations and definitions}

Some efforts have been made to extend the Lie derivative (Isidori (1995)) to nonlinear time-delay systems (see Germani et al. (2001, 2002); Oguchi et al. (2002); Oguchi and Richard (2006); Califano et al. (2011)) in the framework of commutative rings. In what follows we define the derivative and Lie derivative for nonlinear time-delay from the non-commutative point of view.

Let $f(x(t-j \tau))$ and $h(x(t-j \tau))$ for $0 \leq j \leq s$ respectively be an $n$ and $p$ dimensional vector with entries $f_{r} \in \mathcal{K}$ for $1 \leq r \leq n$ and $h_{i} \in \mathcal{K}$ for $1 \leq i \leq p$. Let

$$
\frac{\partial h_{i}}{\partial x}=\left[\frac{\partial h_{i}}{\partial x_{1}}, \cdots, \frac{\partial h_{i}}{\partial x_{n}}\right] \in \mathcal{K}^{1 \times n}(\delta]
$$

where for $1 \leq r \leq n$ :

$$
\frac{\partial h_{i}}{\partial x_{r}}=\sum_{j=0}^{s} \frac{\partial h_{i}}{\partial x_{r}(t-j \tau)} \delta^{j} \in \mathcal{K}(\delta]
$$

then the Lie derivative for nonlinear systems without delays can be extended to nonlinear time-delay systems in the framework of Xia et al. (2002) as follows

$$
L_{f} h_{i}=\frac{\partial h_{i}}{\partial x}(f)=\sum_{r=1}^{n} \sum_{j=0}^{s} \frac{\partial h_{i}}{\partial x_{r}(t-j \tau)} \delta^{j}\left(f_{r}\right)
$$

and in the same way one can define $L_{G_{i}} h_{i}$.

Based on the above notations, the relative degree might be defined in the following way:

Definition 1. (Relative degree) System (6) has relative degree $\left(v_{1}, \cdots, v_{p}\right)$ in an open set $W \subseteq R^{n}$ if, for $1 \leq i \leq p$, the following conditions are satisfied:

1. for all $x \in W, L_{G_{j}} L_{f}^{r} h_{i}=0$, for all $1 \leq j \leq m$ and $0 \leq r<$ $v_{i}-1$;

2. there exists $x \in W$ such that $\exists j \in[1, m], L_{G_{j}} L_{f}^{v_{i}-1} h_{i} \neq 0$.

If the first condition is satisfied for all $r \geq 0$ and $1 \leq i \leq p$, we set $v_{i}=\infty$.

Moreover, for system (6), one can also define observability indices introduced in Krener (1985) over the non-commutative rings. Let $\mathcal{F}_{k}$ be the following left module over $\mathcal{K}(\delta]$ :

$$
\mathcal{F}_{k}:=\operatorname{span}_{\mathcal{K}(\delta]}\left\{d h, d L_{f} h, \cdots, d L_{f}^{k-1} h\right\}
$$

for $1 \leq k \leq n$, and it was shown that the filtration of $\mathcal{K}(\delta]$ module satisfies $\mathcal{F}_{1} \subset \mathcal{F}_{2} \subset \cdots \subset \mathcal{F}_{n}$, then define $d_{1}=$ $\operatorname{rank}_{\mathcal{K}(\delta]} \mathcal{F}_{1}$, and $d_{k}=\operatorname{rank}_{\mathcal{K}(\delta]} \mathcal{F}_{k}-\operatorname{rank}_{\mathcal{K}(\delta]} \mathcal{F}_{k-1}$ for $2 \leq k \leq n$. Let $k_{i}=\operatorname{card}\left\{d_{k} \geq i, 1 \leq k \leq n\right\}$, then $\left(k_{1}, \cdots, k_{p}\right)$ are the observability indices. Reorder, if necessary, the output components of (6), such that

$$
\begin{aligned}
& \operatorname{rank}_{\mathcal{K}(\delta]}\left\{\frac{\partial h_{1}}{\partial x}, \cdots, \frac{\partial L_{f}^{k_{1}-1} h_{1}}{\partial x}, \cdots, \frac{\partial h_{p}}{\partial x}, \cdots, \frac{\partial L_{f}^{k_{p}-1} h_{p}}{\partial x}\right\} \\
& =k_{1}+\cdots+k_{p}
\end{aligned}
$$


Based on the above definitions, let us define the following notations which will be used in the sequel. For $1 \leq i \leq p$, denote $k_{i}$ the observability indices, $v_{i}$ the relative degree for $y_{i}$ of (6), and note

$$
\rho_{i}=\min \left\{v_{i}, k_{i}\right\}
$$

Without loss of generality, suppose $\sum_{i=1}^{p} \rho_{i}=j$, thus $\left\{d h_{1}, \cdots, d L_{f}^{\rho_{1}-1} h_{1}, \cdots, d h_{p}, \cdots, d L_{f}^{\rho_{p}-1} h_{p}\right\}$ are $j$ linearly independent vector over $\mathcal{K}(\delta]$. Then note

$$
\Phi=\left\{d h_{1}, \cdots, d L_{f}^{\rho_{1}-1} h_{1}, \cdots, d h_{p}, \cdots, d L_{f}^{\rho_{p}-1} h_{p}\right\}
$$

and

$$
£=\operatorname{span}_{R[\delta]}\left\{h_{1}, \cdots, L_{f}^{\rho_{1}-1} h_{1}, \cdots, h_{p}, \cdots, L_{f}^{\rho_{p}-1} h_{p}\right\}
$$

where $R[\delta]$ is the commutative ring of polynomials of $\delta$ with coefficients belonging to the field $R$, and let $£(\delta]$ be the set of polynomials of $\delta$ with coefficients over $£$, define the module spanned by element of $\Phi$ over $£(\delta$ ] as follows

$$
\Omega=\operatorname{span}_{\mathfrak{f}(\delta]}\{\xi, \xi \in \Phi\}
$$

Define

$$
\mathcal{G}=\operatorname{span}_{R[\delta]}\left\{G_{1}, \ldots, G_{m}\right\}
$$

where $G_{i}$ is given in (6), and its left annihilator

$$
\mathcal{G}^{\perp}=\operatorname{span}_{\mathfrak{f}(\delta]}\{\omega \in \mathcal{M} \mid \omega \beta=0, \forall \beta \in \mathcal{G}\}
$$

where $\mathcal{M}$ is defined in (7).

\section{Preliminary result}

After having defined the derivative of function belonging to $\mathcal{K}(\delta]$, let us study the delay identification for system (6). The following definition is an adaptation of Definition 2 in Anguelova and Wennberg (2008).

Definition 2. For system (6), an equation

$$
\alpha\left(h, \dot{h} \ldots, h^{(k)}, \delta\right)=0, k \in Z^{+}
$$

is named to be an output delay equation since it contains only the output, its derivatives and delays. Moreover this equation is called to be an output delay-identifiable equation ${ }^{2}$ for (6) if it cannot be written as $\alpha\left(h, \dot{h} \ldots, h^{(k)}, \delta\right)=a(\delta] \tilde{\alpha}\left(h, \dot{h} \ldots, h^{(k)}\right)$ with $a(\delta] \in \mathcal{K}(\delta]$.

As stated in Anguelova and Wennberg (2008), if there exists an output delay-identifiable equation for (6) (i.e. involving the delay in an essential way), then the delay can be identified for almost all $^{3} y$ by numerically finding zeros of such an equation. For this issue, the interested reader can refer to Barbot et al. (2012) and the references therein. Thus delay identification for (6) becomes to seek such an equation.

Let us consider the most simple case for identifying the delay for (6), i.e., from only the outputs of (6), which is stated in the following preliminary result.

\footnotetext{
${ }^{2}$ this equation is stated to involve the delay in an essential way in Anguelova and Wennberg (2008).

${ }^{3}$ singularity of the delay identification exists for a countable set of $y$, which is excluded in this paper.
}

Theorem 1. There exists an output delay-identifiable equation $\alpha(h, \delta)$ for $(6)$ if and only if

$$
\operatorname{rank}_{\mathcal{K}(\delta]} \frac{\partial h}{\partial x}<\operatorname{rank}_{\mathcal{K}} \frac{\partial h}{\partial x}
$$

Proof. Necessity: Suppose that there exists an output delayidentifiable equation which enables to identify the delay of (6), let us show (13) is satisfied. For this, consider the opposite, i.e. assume

$$
\operatorname{rank}_{\mathcal{K}(\delta]} \frac{\partial h}{\partial x}=\operatorname{rank}_{\mathcal{K}} \frac{\partial h}{\partial x},
$$

and this implies that $h$ does not contain any delay, otherwise we have

$$
\operatorname{rank}_{\mathcal{K}(\delta]} \frac{\partial h}{\partial x}<\operatorname{rank}_{\mathcal{K}} \frac{\partial h}{\partial x} .
$$

No involvement of delay in $h$ implies that it is not possible to identify the delay from the output, thus it contradicts the assumption that there exists an output delay-identifiable equation to identify the delay, and we prove the necessity by contradiction.

Sufficiency: Suppose that

$$
\operatorname{rank}_{\mathcal{K}(\delta]} \frac{\partial h}{\partial x}<\operatorname{rank}_{\mathcal{K}} \frac{\partial h}{\partial x} \leq p
$$

then there exists $a_{i} \in \mathcal{K}(\delta]$ for $1 \leq i \leq p$, such that

$$
a_{1} d h_{1}+\cdots+a_{p} d h_{p}=0
$$

The exterior differentiation of the above equation is equal to zero, implying the left side of the above equation is a closed one-form. By applying Poincaré Theorem, there always exists a function $\alpha$ such that

$$
\alpha\left(h_{1}, \ldots, h_{p}, \delta\right)=0
$$

In addition, the above output delay equation should involve $\delta$ in an essential way, because if it is not the case, then $\delta$ can be taken out from $\alpha$, yielding

$$
\operatorname{rank}_{\mathcal{K}(\delta]} \frac{\partial h}{\partial x}=\operatorname{rank}_{\mathcal{K}} \frac{\partial h}{\partial x},
$$

and this contradicts the inequality (13).

Remark 1. Inequality (13) implies that the outputs of (6) are dependent over $\mathcal{K}(\delta]$. Theorem 1 can be seen as a special case of Theorem 2 in Anguelova and Wennberg (2008). However as we will show in the next section that this condition is not necessary for the case where the output of (6) is independent over $\mathcal{K}(\delta]$.

Example 1. Consider the following dynamical system:

$$
\left\{\begin{array}{l}
\dot{x}=f(x, u, \delta) \\
y_{1}=x_{1} \\
y_{2}=x_{1} \delta x_{1}+x_{1}^{2}
\end{array}\right.
$$

It can be seen that

$$
\frac{\partial h}{\partial x}=\left(\begin{array}{ll}
1, & 0 \\
\delta x_{1}+2 x_{1}+x_{1} \delta, & 0
\end{array}\right)
$$


which yields $\operatorname{rank}_{\mathcal{K}(\delta]} \frac{\partial h}{\partial x}=1$ and $\operatorname{rank}_{\mathcal{K}} \frac{\partial h}{\partial x}=2$. Thus Theorem 1 is satisfied, and the delay of system (14) can be identified.

In fact, a straightforward calculation gives

$$
y_{2}=y_{1} \delta y_{1}+y_{1}^{2}
$$

which permits to identify the delay $\delta$ by applying an algorithm to detect zero-crossing when varying $\delta$.

\section{Main result}

Identification of delay for (6) from its output is analyzed in the last section, this section considers the case where the output of (6) is independent over $\mathcal{K}(\delta]$.

Since for (6) we denoted $k_{i}$ the observability indices and $v_{i}$ the relative degree for $y_{i}$ of $(6)$, and noted $\rho_{i}=\min \left\{v_{i}, k_{i}\right\}$ for $1 \leq i \leq p$, then one obtains

$$
\mathcal{H}(x, \delta)=\Psi(x, \delta)+\Gamma(x, \delta) u
$$

with

$$
\begin{aligned}
& \mathcal{H}(x, \delta)=\left(h_{1}^{\left(\rho_{1}\right)}, \cdots, h_{p}^{\left(\rho_{p}\right)}\right)^{T} \\
& \Psi(x, \delta)=\left(L_{f}^{\rho_{1}} h_{1}, \cdots, L_{f}^{\rho_{p}} h_{p}\right)^{T}
\end{aligned}
$$

and

$$
\Gamma(x, \delta)=\left(\begin{array}{ccc}
L_{G_{1}} L_{f}^{\rho_{1}-1} h_{1} & \cdots & L_{G_{m}} L_{f}^{\rho_{1}-1} h_{1} \\
\vdots & \ddots & \vdots \\
L_{G_{1}} L_{f}^{\rho_{p}-1} h_{p} & \cdots & L_{G_{m}} L_{f}^{\rho_{p}-1} h_{p}
\end{array}\right)
$$

Based on the above definitions, we are ready to state our main result.

Theorem 2. There exists an output delay equation for (6), if there exists a non zero $\omega=\sum_{c=1}^{n} \sum_{j=1}^{p} q_{j} \frac{\partial L_{f}^{\rho_{j}-1} h_{j}}{\partial x_{c}} d x_{c}$ with $q_{j} \in$ $\mathcal{K}(\delta]$ for $1 \leq j \leq p$, such that $\omega \in \mathcal{G}^{\perp} \cap \Omega$ and $\omega f \in £$, where $\mathcal{G}^{\perp}$ is defined in (12), $\Omega$ is defined in (11), and $£$ is defined in (10).

Proof. Denote $Q=\left[q_{1}, \cdots, q_{p}\right]$ be $1 \times p$ vector with $q_{j} \in \mathcal{K}(\delta]$ for $1 \leq j \leq p$. One has

$$
\begin{aligned}
Q \Gamma & =Q\left(\begin{array}{ccc}
L_{G_{1}} L_{f}^{\rho_{1}-1} h_{1} & \cdots & L_{G_{m}} L_{f}^{\rho_{1}-1} h_{1} \\
\vdots & \ddots & \vdots \\
L_{G_{1}} L_{f}^{\rho_{p}-1} h_{p} & \cdots & L_{G_{m}} L_{f}^{\rho_{p}-1} h_{p}
\end{array}\right) \\
& =\left(Q\left[\begin{array}{c}
\frac{\partial L_{f}^{\rho_{1}-1} h_{1}}{\partial x} \\
\vdots \\
\frac{\partial L_{f}^{\rho_{p}-1} h_{p}}{\partial x}
\end{array}\right]\right)\left[G_{1}, \cdots, G_{m}\right]
\end{aligned}
$$

because of the associativity law over $\mathcal{K}(\delta]$. Then according to the definition (8), one gets

$$
Q \Gamma=\omega\left[G_{1}, \cdots, G_{m}\right]=\omega G
$$

where $\omega=\sum_{c=1}^{n} \sum_{j=1}^{p} q_{j} \frac{\partial L_{f}^{\rho_{j}-1} h_{j}}{\partial x_{c}} d x_{c}$.
Moreover, it is easy to check that

$$
\begin{aligned}
\omega f & =\left(Q\left[\begin{array}{c}
\frac{\partial L_{f}^{\rho_{1}-1} h_{1}}{\partial x} \\
\vdots \\
\frac{\partial L_{f}^{\rho_{p}-1} h_{p}}{\partial x}
\end{array}\right]\right) f=Q\left(\left[\begin{array}{c}
\frac{\partial L_{f}^{\rho_{1}-1} h_{1}}{\partial x} \\
\vdots \\
\frac{\partial L_{f}^{\rho_{p}-1} h_{p}}{\partial x}
\end{array}\right] f\right) \\
& =Q\left[\begin{array}{c}
L_{f}^{\rho_{1}} h_{1} \\
\vdots \\
L_{f}^{\rho_{p}} h_{p}
\end{array}\right]=Q \Psi
\end{aligned}
$$

According to (15), one has

$$
Q \mathcal{H}=Q(\Psi+\Gamma u)=\omega f+\omega G u
$$

where $\mathcal{H}=\left[y_{1}^{\left(\rho_{1}\right)}, \cdots, y_{p}^{\left(\rho_{p}\right)}\right]^{T}$ is a vector which can be estimated in finite time.

Thus, if $\omega \in \mathcal{G}^{\perp} \cap \Omega$ and $\omega f \in \mathfrak{E}$, which implies there exists $Q$ with entries belonging to $£(\delta]$, one has

$$
Q \Gamma=\omega G=0
$$

and

$$
Q \mathcal{H}=\omega f \in \mathfrak{E}
$$

Finally one obtains the following relation:

$$
Q(\mathcal{H}-\Psi)=0
$$

which contains only the output, its derivatives and delays.

Obviously, if (18) is an output delay-identifiable equation, i.e. containing the delay $\delta$ in an essential way, then the delay of (6) can be identified by detecting zero-crossing of (18). Before to give necessary and sufficient conditions guaranteeing the essential involvement of $\delta$ in (18), define

$$
\mathcal{Y}=\left(h_{1}, \ldots, L_{f}^{\rho_{1}-1} h_{1}, \ldots, h_{p}, \ldots, L_{f}^{\rho_{p}-1} h_{p}\right)^{T}
$$

and denote $\mathcal{K}_{0} \subset \mathcal{K}$ the field of meromorphic functions of $x$, which will be used in the following theorem.

Theorem 3. The output delay equation (18) is an output delayidentifiable equation if and only if

$$
\operatorname{rank}_{\mathcal{K}(\delta]} \frac{\partial \mathcal{Y}}{\partial x}<\operatorname{rank}_{\mathcal{K}} \frac{\partial\{\mathcal{Y}, \Psi\}}{\partial x}
$$

or for any element $q_{j}$ of $Q \in \mathcal{K}^{1 \times p}(\delta], \nexists a(\delta] \in \mathcal{K}(\delta]$ such that

$$
q_{j}=a(\delta] \bar{q}_{j} \text {, with } \bar{q}_{j} \in \mathcal{K}_{0}, 1 \leq j \leq p
$$

and

$$
\operatorname{rank}_{\mathcal{K}(\delta]} \frac{\partial \mathcal{Y}}{\partial x}=\operatorname{rank}_{\mathcal{K}} \frac{\partial\{\mathcal{Y}, \Psi\}}{\partial x}
$$

Proof. Necessity: Suppose that (18) involves the delay $\delta$ in an essential way, we will show that either (19) or (20-21) is satisfied. We prove this by contradiction and let us consider the opposite of (19-21), i.e.

$$
\operatorname{rank}_{\mathcal{K}(\delta]} \frac{\partial \mathcal{Y}}{\partial x}=\operatorname{rank}_{\mathcal{K}} \frac{\partial\{\mathcal{Y}, \Psi\}}{\partial x}
$$


and

$$
q_{j}=a(\delta] \bar{q}_{j} \text { with } \bar{q}_{j} \in \mathcal{K}_{0}, 1 \leq j \leq p
$$

for all $q_{j} \in \mathcal{K}(\delta]$ in $Q$.

It can be seen that (22) implies that $\Psi$ is a function of $y$ without $\delta$, and (23) implies that there exists a scalar $a(\delta] \in \mathcal{K}(\delta]$ such that

$$
Q=a(\delta] \bar{Q},
$$

where $\bar{Q}$ is the associated vector with entries belonging to the field $\mathcal{K}_{0}$, then

$$
Q(\mathcal{H}-\Psi)=a(\delta](\bar{Q}(\mathcal{H}-\Psi))=0,
$$

and this contradicts the assumption that (18) involves the delay $\delta$ in an essential way, so the necessity is proven by contradiction.

Sufficiency: Firstly, suppose that

$$
\operatorname{rank}_{\mathcal{K}(\delta]} \frac{\partial \mathcal{Y}}{\partial x}<\operatorname{rank}_{\mathcal{K}} \frac{\partial\{\mathcal{Y}, \Psi\}}{\partial x},
$$

then $\Psi$ should be a function of $\mathcal{Y}$ containing $\delta$ in an essential way, since if it is not the case, one can find a function $\alpha$ such that

$$
\alpha(\Psi, \mathcal{Y})=0,
$$

which implies that

$$
\operatorname{rank}_{\mathcal{K}} \frac{\partial\{\mathcal{Y}, \Psi\}}{\partial x} \leq \operatorname{rank}_{\mathcal{K}} \frac{\partial\{\mathcal{Y}\}}{\partial x}=\operatorname{rank}_{\mathcal{K}(\delta]} \frac{\partial \mathcal{Y}}{\partial x},
$$

since $\mathcal{Y}$ is linearly independent over $\mathcal{K}(\delta]$. Because $\Psi$ is a function of $\mathcal{Y}$ containing $\delta$ in an essential way, thus for any $Q$, the function $Q(\mathcal{H}-\Psi)=0$ always contains $\delta$ in an essential way.

Secondly, since

$$
\operatorname{rank}_{\mathcal{K}(\delta]} \frac{\partial \mathcal{Y}}{\partial x} \leq \operatorname{rank}_{\mathcal{K}} \frac{\partial\{\mathcal{Y}\}}{\partial x} \leq \operatorname{rank}_{\mathcal{K}} \frac{\partial\{\mathcal{Y}, \Psi\}}{\partial x}
$$

and

$$
\operatorname{rank}_{\mathcal{K}(\delta]} \frac{\partial \mathcal{Y}}{\partial x} \leq \operatorname{rank}_{\mathcal{K}(\delta]} \frac{\partial\{\mathcal{Y}, \Psi\}}{\partial x} \leq \operatorname{rank}_{\mathcal{K}} \frac{\partial\{\mathcal{Y}, \Psi\}}{\partial x},
$$

so if

$$
\operatorname{rank}_{\mathcal{K}(\delta]} \frac{\partial \mathcal{Y}}{\partial x}=\operatorname{rank}_{\mathcal{K}} \frac{\partial\{\mathcal{Y}, \Psi\}}{\partial x},
$$

then one obtains

$$
\operatorname{rank}_{\mathcal{K}} \frac{\partial\{\mathcal{Y}\}}{\partial x}=\operatorname{rank}_{\mathcal{K}} \frac{\partial\{\mathcal{Y}, \Psi\}}{\partial x}
$$

and

$$
\operatorname{rank}_{\mathcal{K}(\delta]} \frac{\partial \mathcal{Y}}{\partial x}=\operatorname{rank}_{\mathcal{K}(\delta]} \frac{\partial\{\mathcal{Y}, \Psi\}}{\partial x} .
$$

The above two equalities imply that $\Psi$ should be a function of $y$ without delays, otherwise one has

$$
\operatorname{rank}_{\mathcal{K}(\delta]} \frac{\partial \mathcal{Y}}{\partial x} \neq \operatorname{rank}_{\mathcal{K}} \frac{\partial\{\mathcal{Y}, \Psi\}}{\partial x},
$$

and this contradicts the assumption of the satisfactory of (21).
Moreover, if there does not exist an $a(\delta] \in \mathcal{K}(\delta]$ such that $q_{j}=a(\delta] \bar{q}_{j}$ for any element $q_{j}$ in $Q$ and $\bar{q}_{j} \in \mathcal{K}_{0}$, then it implies that $Q$ contains delays in an essential way. Otherwise one can find a vector $\bar{Q}$ with entries belonging to $\mathcal{K}_{0}$ and an $a(\delta] \in \mathcal{K}(\delta]$ such that $Q=a(\delta] \bar{Q}$, which implies $Q$ does not contain delays in an essential way.

Finally the essential involvement of $\delta$ in $Q$ means the essential involvement of $\delta$ in the function:

$$
Q(\mathcal{H}-\Psi)=0
$$

even when $\Psi$ does not contain any delay.

Remark 2. It is clear that Theorem 1 is a special case of Theorem 3 , since the output delay-identifiable equation stated in Theorem 1 does not contain any derivative of the output.

A similar condition as (19) of Theorem 3 is stated as a necessary and sufficient condition for identifying delay for nonlinear time-delay systems with known inputs in Anguelova and Wennberg (2008). However as we proved above that it is only sufficient, but not necessary for the case with unknown inputs.

\section{Application to JAK-STAT}

In order to highlight the proposed results, let us consider the dynamics of the JAK-STAT (JAnus Kinase - Signal Transducer and Activator of Transcription) signaling pathway in the cell from Timmer and Muller (2004).

The JAK-STAT signaling pathway transmits information from chemical signals outside of the cell, through the cell membrane, and into gene promoters on the DNA in the cell nucleus, which causes DNA transcription and activity in the cell.

Binding of the hormone erythropoietin (Epo) to the extracellular part of the receptor leads to activation by phosphorylation of the so-called JAK at intracellular, cytoplasmic domain of the receptor. In trun, this leads to phosphorylation of monomeric STAT-5 forms dimers and these dimers migrate into the nucleus where they bind to promotor region of the DNA and initiate gene transcription.

Denote the amount of activated Epo-receptors by $u$, unphosphorylated monomeric STAT-5 by $x_{1}$, phosphorylated monomeric STAT- 5 by $x_{2}$, phosphorylated dimeric STAT- 5 in the cytoplasm by $x_{3}$ and phosphorylated dimeric STAT-5 in the nucleus by $x_{4}$, the STAT- 5 cycling model can be described as follows:

$$
\left\{\begin{array}{l}
\dot{x}_{1}=-k_{1} x_{1} u+2 k_{4} \delta x_{3} \\
\dot{x}_{2}=k_{1} x_{1} u-k_{2} x_{2}^{2} \\
\dot{x}_{3}=-k_{3} x_{3}+k_{2} x_{2}^{2} / 2 \\
\dot{x}_{4}=k_{3} x_{3}-k_{4} \delta x_{3} \\
y_{1}=x_{1} \\
y_{2}=x_{2} \\
y_{3}=x_{4}
\end{array}\right.
$$

In the above JAK-STAT model, the coefficients $k_{1}-k_{4}$ are known constants. Note that $u$ representing the amount of activated Epo-receptors is an external input, thus it is assumed to be unknown in this paper.We are going to identify the delay $\delta$ in (24). 
For the outputs of (24), one has

$$
k_{1}=v_{1}=1, k_{2}=v_{2}=1
$$

and

$$
k_{3}=2, v_{3}=3
$$

which gives $\rho_{1}=1, \rho_{2}=1$ and $\rho_{3}=2$. Then one obtains

$$
\Phi=\left\{d x_{1}, d x_{2}, d x_{4},\left(k_{3}-k_{4} \delta\right) d x_{3}\right\}
$$

By simple calculations, one obtains

$$
\begin{aligned}
£ & =\operatorname{span}_{R[\delta]}\left\{x_{1}, x_{2}, x_{4},\left(k_{3}-k_{4} \delta\right) x_{3}\right\} \\
\mathcal{G}^{\perp} & =\operatorname{span}_{\mathfrak{f}(\delta]}\left\{d x_{1}+d x_{2}, d x_{3}, d x_{4}\right\}
\end{aligned}
$$

and

$$
\Omega=\operatorname{span}_{\mathfrak{f}(\delta]}\left\{d x_{1}, d x_{2}, d x_{4},\left(k_{3}-k_{4} \delta\right) d x_{3}\right\}
$$

which gives

$$
\begin{aligned}
\Omega \cap \mathcal{G}^{\perp}= & \operatorname{span}_{\mathfrak{f}(\delta]}\left\{d x_{1}, d x_{2}, d x_{4},\left(k_{3}-k_{4} \delta\right) d x_{3}\right\} \\
& \cap \operatorname{span}_{\mathfrak{f}(\delta]}\left\{d x_{1}+d x_{2}, d x_{3}, d x_{4}\right\} \\
= & \operatorname{span}_{\mathfrak{f}(\delta]}\left\{d x_{1}+d x_{2},\left(k_{3}-k_{4} \delta\right) d x_{3}, d x_{4}\right\}
\end{aligned}
$$

By choosing $Q=(0,0,1)$, a non zero one-form can be found, such as

$$
\omega=\left(k_{3}-k_{4} \delta\right) d x_{3} \in \Omega \cap \mathcal{G}^{\perp}
$$

satisfying

$$
\omega f=-k_{3}\left(k_{3}-k_{4} \delta\right) x_{3}+k_{2}\left(k_{3}-k_{4} \delta\right) x_{2}^{2} / 2 \in £
$$

Thus the following function

$$
Q(\mathcal{H}-\Psi)=0
$$

contains only the output, its derivatives and delays, where

$$
\begin{gathered}
\mathcal{H}=\left(\dot{y}_{1}, \dot{y}_{2}, \ddot{y}_{3}\right)^{T} \\
\Psi=\left(2 k_{4} \delta x_{3},-k_{2} x_{2}^{2},\left(k_{3}-k_{4} \delta\right)\left(-k_{3} x_{3}+k_{2} x_{2}^{2} / 2\right)\right)^{T}
\end{gathered}
$$

Since $\boldsymbol{y}=\left(x_{1}, x_{2}, x_{4},\left(k_{3}-k_{4} \delta\right) x_{3}\right)^{T}$, one has

$$
\frac{\partial \mathcal{Y}}{\partial x}=\left(\begin{array}{cccc}
1, & 0, & 0, & 0 \\
0, & 1, & 0, & 0 \\
0, & 0, & 0, & 1 \\
0, & 0, & k_{3}-k_{4} \delta, & 0
\end{array}\right)
$$

and

$$
\frac{\partial \Psi}{\partial x}=\left(\begin{array}{cccc}
0, & 0, & 2 k_{4} \delta, & 0 \\
0, & -2 k_{2} x_{2}, & 0, & 0 \\
0, & k_{2}\left(k_{3}-k_{4} \delta\right) x_{2}, & -k_{3}\left(k_{3}-k_{4} \delta\right), & 0
\end{array}\right)
$$

Thus, one obtains

$$
\operatorname{rank}_{\mathcal{K}(\delta]} \frac{\partial \mathcal{Y}}{\partial x}=4<\operatorname{rank}_{\mathcal{K}} \frac{\partial\{\mathcal{Y}, \Psi\}}{\partial x}=6
$$

then Theorem 3 is satisfied and (25) involves $\delta$ in an essential way. A straightforward calculation gives

$$
\ddot{y}_{3}=-k_{3} \dot{y}_{3}+k_{2} k_{3} y_{2}^{2} / 2-k_{2} k_{4}\left(\delta y_{2}\right)^{2} / 2
$$

which permits to identify $\delta$.

Let us remark that the choice of non zero one-form $\omega$ is not unique, which leads to different output delay-identifiable equations. For example, by choosing

$$
Q=\left[k_{3}\left(k_{3}-k_{4} \delta\right), k_{3}\left(k_{3}-k_{4} \delta\right), 2 k_{4} \delta\right]
$$

one obtains

$$
\omega=k_{3}\left(k_{3}-k_{4} \delta\right) d x_{1}+k_{3}\left(k_{3}-k_{4} \delta\right) d x_{2}+2 k_{4} \delta\left(k_{3}-k_{4} \delta\right) d x_{3}
$$

such that

$$
\omega f=-k_{2}\left(k_{3}-k_{4} \delta\right)^{2} x_{2}^{2} \in £
$$

Based on this new $Q$, for the output delay-identifiable equation $Q(\mathcal{H}-\Psi)=\omega f-Q \Psi=0$, one obtains

$$
\begin{aligned}
k_{3}\left(k_{3}-k_{4} \delta\right)\left(\dot{y}_{1}+\dot{y}_{2}\right)+2 k_{4} \delta \ddot{y}_{3} & =-k_{2}\left(k_{3}-k_{4} \delta\right)^{2} x_{2}^{2} \\
& =-k_{2}\left(k_{3}-k_{4} \delta\right)^{2} y_{2}^{2}
\end{aligned}
$$

which can be used as well to identify $\delta$.

\section{Conclusion}

This paper deals with the delay identification of time-delay systems with unknown inputs. Necessary and sufficient condition for the most simple case, i.e. identification of the delay directly from the output, has been studied. For a more generic case, sufficient condition is given in order to guarantee the existence of an output delay equation. Necessary and sufficient condition is then discussed to check whether the deduced output delay equation can be used to identify the delay. The obtained results are valid for the systems with single delay, and the derived necessary and sufficient conditions become only necessary for the systems with multi delays.

Anguelova, M., Wennberg, B., 2008. State elimination and identifiability of the delay parameter for nonlinear time-delay systems. Automatica 44 (5), 1373-1378.

Barbot, J.-P., Zheng, G., Floquet, T., Boutat, D., Richard, J.-P., 2012. Delay estimation algorithm for nonlinear time-delay systems with unknown inputs. in Proc. of IFAC Workshop on Time Delay Systems.

Belkoura, L., 2005. Identifiability of systems described by convolution equations. Automatica 41 (3), 505-512.

Belkoura, L., Richard, J.-P., Fliess, M., 2009. Parameters estimation of systems with delayed and structured entries. Automatica 45 (5), 1117-1125.

Califano, C., Marquez-Martinez, L., Moog, C., 2011. Extended lie brackets for nonlinear time-delay systems. IEEE Transactions on Automatic Control 56 (9), 2213-2218.

Conte, G., Moog, C., Perdon, A., 1999. Nonlinear control systems: An algebraic setting. Lecture Notes in Control and Information Sciences 242, Springer-Verlag,London

Darouach, M., Zasadzinski, M., Xu, S., 1994. Full order observers for linear systems with unknown inputs. IEEE Transaction on Automatic Control 39 (3), 606-609.

Drakunov, S., Perruquetti, W., Richard, J.-P., Belkoura, L., 2006. Delay identification in time-delay systems using variable structure observers. Annual Reviews in Control 30 (2), 143-158.

Fliess, M., Sira-Ramirez, H., 2004. Reconstructeurs d'état. Comptes Rendus de 1'Académie des Sciences - Series I 338 (1), 91-96.

Germani, A., Manes, C., Pepe, P., 2001. An asymptotic state observer for a class of nonlinear delay systems. Kybernetika 37 (4), 459-478.

Germani, A., Manes, C., Pepe, P., 2002. A new approach to state observation of nonlinear systems with delayed output. IEEE Transactions on Automatic Control 47 (1), 96-101. 
Isidori, A., 1995. Nonlinear control systems (3rd edition). London: SpringerVerlag.

Ježek, J., 1996. Rings of skew polynomials in algebraical approach to control theory. Kybernetika 32 (1), 63-80.

Koenig, D., Bedjaoui, N., Litrico, X., 2005. Unknown input observers design for time-delay systems application to an open-channel. in Proc. of IEEE Conference on Decision and Control.

Kolmanovskii, V., Myshkis, A., 1999. Introduction to the theory and application of functional differential equations. Kluwer Academic Publishers, Dordrecht.

Krener, A., 1985. (A $\left.d_{f, g}\right),\left(a d_{f, g}\right)$ and locally $\left(a d_{f, g}\right)$ invariant and controllability distributions. SIAM Journal on Control and Optimization 23 (4), 523549

Moog, C., Castro-Linares, R., Velasco-Villa, M., Marque-Martinez, L. A., 2000. The disturbance decoupling problem for time-delay nonlinear systems. IEEE Transactions on Automatic Control 45 (2), 305-309.

Niculescu, S.-I., 2001. Delay effects on stability: A robust control approach Lecture Notes in Control and Information Sciences, Springer 269.

Oguchi, T., Richard, J.-P., 2006. Sliding-mode control of retarded nonlinear systems via finite spectrum assignment approach. IEEE Transactions on Automatic Control 51 (9), 1527-1531.

Oguchi, T., Watanabe, A., Nakamizo, T., 2002. Input-output linearization of retarded non-linear systems by using an extension of lie derivative. International Journal of Control 75 (8), 582-590.

Ren, X., Rad, A., Chan, P., Lo, W., 2005. Online identification of continuoustime systems with unknown time delay. IEEE Transaction on Automatic Control 50 (9), 1418-1422.

Richard, J.-P., 2003. Time-delay systems: an overview of some recent advances and open problems. Automatica 39 (10), 1667-1694.

Sename, O., 1994. Unknown input robust observers for time-delay systems. in Proc. of IEEE Conference on Decision and Control.

Timmer, J., Muller, T., 2004. Modeling the nonlinear dynamics of cellular signal transduction. International Journal of Bifurcation and Chaos 14 (6), 2069-2079.

Xia, X., Marquez, L., Zagalak, P., Moog, C., 2002. Analysis of nonlinear time-delay systems using modules over non-commutative rings. Automatica 38 (9), 1549-1555.

Yang, F., Wilde, R., 1988. Observers for linear systems with unknown inputs. IEEE Transactions on Automatic Control 33 (7), 677-681.

Zheng, G., Barbot, J.-P., Floquet, T., Boutat, D., Richard, J.-P., 2011. On obserability of nonlinear time-delay systems with unknown inputs. IEEE Transactions on Automatic Control 56 (8), 1973-1978. 